\title{
Erratum
}

\section{Level and Pythagoras number of some geometric rings}

\section{Louis Mahé}

IRMAR, Campus de Beaulieu, F-35042 Rennes-Cedex, France

Math. Z. 204, 615-629 (1990)

Statements 1) and 3) of Theorem 4.1 are false. The correct statement of the theorem is the following

Theorem 4.1 Let $A$ be a finitely generated $R$-algebra of Krull dimension $\leqq d$, and $f$ a totally positive element of $A$. Then

1) if $d \leqq 3$, we have an identity

$$
f 2^{d+1}-1=1+2^{d+1}-1
$$

2) if $d \leqq 4$ and $f$ is a unit in $A$, we have

$$
f 2^{d}=1+2^{d}-1
$$

3) if $d \geqq 4$, we have

$$
f 2^{d}+7=1+2^{d+1}+d-4
$$

4) if $d \geqq 5$ and $f$ is a unit in $A$, we have

$$
f 2^{d}=1+2^{d}+d-5 .
$$

Subsequently, Corollary 4.4 on the level has to be modified as follows:

Corollary 4.4 Let $A$ be an $R$-algebra of transcendence degree $d$ without any real point (i.e. $\mathrm{Spec}_{\mathrm{r}} A=\emptyset$ ), then

1) if $d \leqq 4$ then $-1=2^{d+1}-1$

2) if $d \geqq 5$ then $-1=2^{d+1}+d-5$.

Proof. Apply 2) or 4) of Proposition 4.3 to $f=-1$ which is a totally positive unit when $\operatorname{Spec}_{r} A=\emptyset$.

Also in Remark 4.5, if the level of a curve without any real point is actually less than 3 (because $2^{1}+1=2^{2}-1$ !), the real bound for surfaces is 7 instead of 5 . Nothing else in the paper is affected by this modification, except the proof of Theorem 4.1, which is the subject of Sect. 5. 
We give below a complete replacement of the end of Sect. 5 starting from line 18 p. 624.

Let us prove part 1). Let $d=\operatorname{tr} . d .(A)$. If $f$ is not a unit, it may be 0 in the function field $K_{i}$ of level $2^{d}$, of some complex irreducible component of $A$ and in that case, we get $0=1+2^{d}$. Because 0 has the shape $f 2^{d-1}$, we can

write $f 2^{d-1}=1+2^{d}$ in every field $K_{i}$, and thus in the product $\prod K_{i}$.

In the 0 -dimensional case, we have $f[1]=1+1$ in each $K_{i}$, and so in $A$.

In dimension 1, with the above arguments, we obtain an identity

$$
\left(1+1-f[1)^{2}+2-f[1=0\right.
$$

in $A$. Applying Lemma 3.1 with $X=1$ and $Y=[1-f[1$, we get

$$
1+[-f[1]+(1+[1-f[1](2-f[1)=0
$$

which gives

$$
1+1-f[1+2-f[2]=0
$$

and eventually

$$
f[3]=1+3 .
$$

The same method in dimension 2 , starting from

$$
\left(1+3-f[3)^{2}+4-f[2]=0\right.
$$

leads (using Lemma 3.1 and Lemma 3.3 part 1) to

$$
f[7=1+7 .
$$

For the dimension 3, we do as in dimension 4 when $f$ is a unit, starting with a double reduction in the dimension to get an identity

$$
\left(\left(1+3-f[3)^{2}+4-f[2)^{2}+8-f[4]=0\right.\right.
$$

and using Lemmas 3.1 with $X=\left(1+3-f[3)^{2}\right.$ and $Y=4-f[2$, we get

$$
\left(1+[3]-f[3)^{6}+[4]-f[2]+(5-f[2])([8]-f[4)=0\right.
$$

With Lemmas $3.2(n=6)$ and 3.3 part 2 , this gives

$$
\left(1+3-f[3)^{2}+4-f[2]+8[8=0\right.
$$

and a last application of Lemma 3.1 gives

$$
1+3-f[3+4-f[4+8-f[8]=1+15-f[15=0
$$

In order to get the parts 3) and 4) of Theorem 4.1, we make an induction respectively for $d \geqq 3$ and $d \geqq 4$. Let tr.d. $(A)=d+1$ and $f$ a totally positive (resp. unit) in $A$. Letting $d^{\prime}=d+1$ (resp. $d$ ), we have

$$
g^{2}+2^{d^{d}}-f 2^{d}=0
$$


in $A$ for some non zero-divisor $g$. By induction hypothesis, we get

$$
1+s_{d}-\bar{f} t_{d}=0
$$

in $A / g$ for $s_{d}=2^{d+1}+d-4$ (resp. $\left.s_{d}=2^{d}+d-5\right)$ and $t_{d}=2^{d}+7$ (resp. $2^{d}$ ), and so

$$
\lambda g=1+s_{d}-f t_{d}
$$

in $A$, and

$$
(\lambda g)^{2}=(1+x-f y)^{2}
$$

where $x=s_{d}$ and $y=t_{d}$. This gives

$$
(\lambda g)^{2}=1+(x-f y)^{2}+2(x-f y)
$$

But because 2 is a square, we get

$$
(\lambda g)^{2}=1+s_{d}+1-f\left[t_{d} .\right.
$$

Returning to the original equation, we get

$$
1+\left[s_{d}+1-f\left[t_{d}\right]+2^{d^{\prime}}-f\left[2^{\bar{d}}\right]=1+\overline{s_{d}+1+2^{d}}-f\left[t_{d}\right]+\left[2^{d} \mid=0 .\right.\right.
$$

In the non unit case, putting $s_{3}=15=2^{4}+3-4, d^{\prime}=d+1$ and $t_{3}=15=2^{3}+7$, the induction relations $s_{d+1}=1+s_{d}+2^{d+1}$ and $t_{d+1}=t_{d}+2^{d}$ give the result.

In the unit case, putting $s_{4}=15=2^{4}+4-5$ and $t_{4}=16=2^{4}$, the relations $s_{d+1}=1+s_{d}+2^{d}$ and $t_{d+1}=t_{d}+2^{d}$ give the result. 